\title{
Tratamiento de cáncer de pulmón metastásico (estadio IV) de célula no pequeña. Consenso de expertos, Asociación Colombiana de Hematología y Oncología (ACHO)
}

Treatment of non-small cell metastatic lung cancer (stage IV). Expert consensus, Colombian Association of Hematology and Oncology (ACHO)

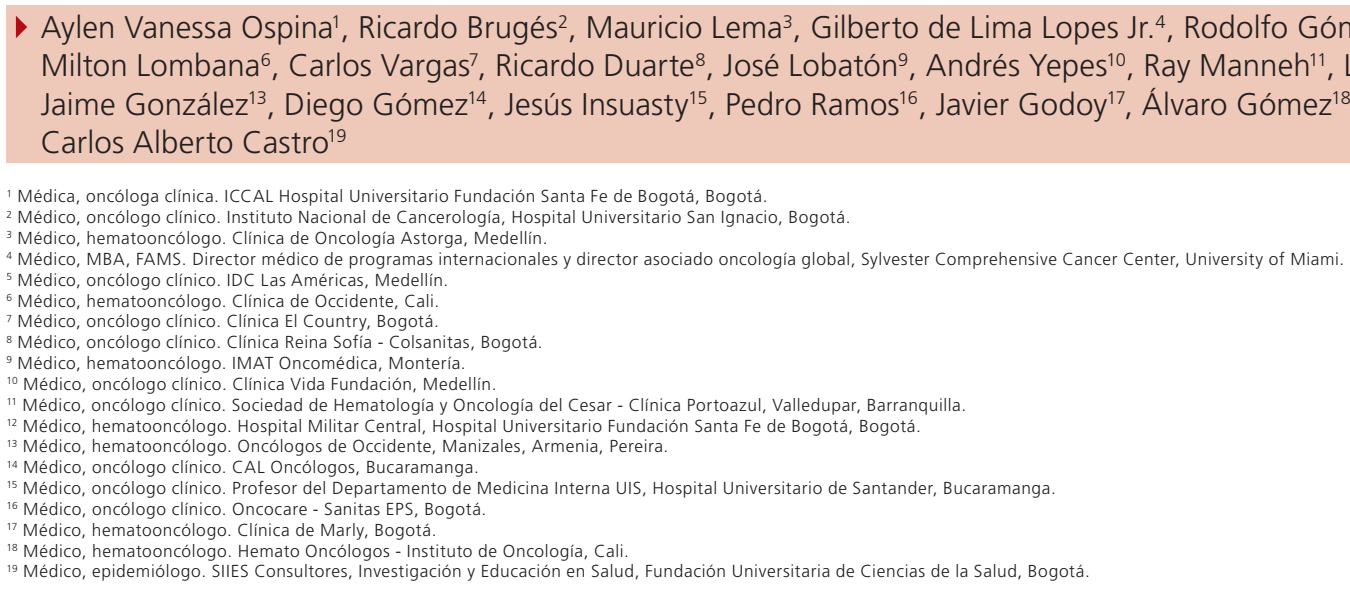

\section{Resumen}

Introducción: el cáncer de pulmón (CP) es la causa más frecuente de muertes por patologías oncológicas en el mundo1, reportando tasas de incidencia para ambos sexos de 23,1 casos por cada 100 mil habitantes y una mortalidad del 19,4\%, según Globocan $2012^{2}$.

Objetivo: consensuar por común acuerdo de expertos recomendaciones para el manejo del cáncer de pulmón metastásico (estadio IV) de célula no pequeña en el contexto colombiano.

Métodos: se estructuró un consenso de expertos constituido por 17 oncólogos clínicos, miembros activos de la Asociación Colombiana de Hematología y Oncología (ACHO), que laboran en las principales instituciones del país. Se llevó a cabo en cuatro fases: en la primera fase, se definieron 22 preguntas, las cuales fueron calificadas de 1 a 9; en la segunda, se reenviaron de nuevo las preguntas no consensuadas; en la tercera, se analizaron y discutieron las respuestas; y las no consensuadas fueron a una cuarta fase, donde se hizo un consenso nominal.

Resultados: se evaluaron y discutieron 25 preguntas relacionadas con el tratamiento de cáncer de pulmón metastásico (estadio IV) de célula no pequeña y se concluyeron recomendaciones basadas en la mejor evidencia científica y en guías de manejo de oncología reconocidas internacionalmente adaptadas al contexto y realidad colombiana.

Conclusiones: se deben considerar los tratamientos para el cáncer de pulmón avanzado según la mejor evidencia científica y la disponibilidad en Colombia, con el fin de ofrecer el mejor tratamiento al paciente. Este consenso es una herramienta para orientar la toma de decisiones clínicas y es útil como documento soporte para consultas de entes públicos y privados. Es importante mencionar que este es un texto académico-científico y no regulatorio.

Palabras clave (DeCS): neoplasias pulmonares, carcinoma de pulmón de célula no pequeña, tratamiento.

Recibido el 14 de julio de 2017; aceptado el 30 de agosto de 2017 


\begin{abstract}
Introduction: Lung cancer (LC) is the most frequent cause of death from oncological diseases in the world ${ }^{1}$, reporting worldwide incidence rates for both sexes of 23.1 cases per 100,000 inhabitants and a mortality rate of $19.4 \%$ according to Globocan $2012^{2}$.

Objective: To agree upon recommendations for the management of non-small cell metastatic lung cancer (stage IV), in the Colombian context.

Methods: A consensus of experts comprised by 17 clinical oncologists, active members of the Colombian Association of Hematology and Oncology (ACHO), who work in the main institutions of the country. It was carried out in four phases: in the first phase 22 questions were structured, which were rated from 1 to 9 , in the second phase the non-consensual questions were re-sent, in the third phase the answers and the answers were analyzed and discussed. The non-consensual ones went to a fourth phase where a nominal consensus was made.

Results: 25 questions related to the treatment of non-small cell metastatic lung cancer (stage IV) were evaluated and conclusions were reached based on the best scientific evidence and internationally recognized oncology management guides adapted to the Colombian context and reality.

Conclusions: Treatments for advanced lung cancer should be considered according to the best scientific evidence and availability in Colombia, in order to offer the best treatment to the patient. This consensus is a tool to guide clinical decision making and is useful as a support document for consultations with public and private entities. It is important to mention that this is an academic-scientific and non-regulatory document.
\end{abstract}

Key words (MeSH): Lung neoplasms, carcinoma, non-small-cell lung, therapeutics.

\section{Introducción}

El cáncer de pulmón (CP) es la causa más frecuente de muertes por patologías oncológicas en el mundo', reportando tasas de incidencia para ambos sexos de 23,1 casos por cada 100 mil habitantes y una mortalidad del 19,4\%, según Globocan 2012², la cual se asemeja a la suma de muertes producidas por cáncer de páncreas, próstata, mama y colon. Se han estimado 234.030 casos incidentes y 154.050 muertes por cáncer de pulmón en los Estados Unidos, de acuerdo con el Instituto Nacional de Salud ${ }^{3}$, siendo más frecuente en hombres que en mujeres y más prevalente por encima de los 65 años. La tasa de sobrevida al año se ha estimado en el $44 \%$ y a cinco años en el $17 \%$; esto depende de múltiples factores, como la clasificación, el estadio del tumor y la presencia de metástasis. Para el caso del $C P$ de células no pequeñas en estadios IA y IB, se han calculado tasas de sobrevida a cinco años del 49\%; para los estadios IIA y IIB, entre el 30\% y el 31\%; sin embargo, cuando hay presencia de metástasis (estadio IV), la sobrevida disminuye al $1 \%$ a cinco años ${ }^{4}$. Para Latinoamérica, conforme con Globocan, los valores son similares a los datos mundiales con una incidencia de $21,6 \times 100$ mil habitantes y una mortalidad de 20,4 x 100 mil habitantes. En Colombia, el cáncer de pulmón es la segunda causa de muerte en hombres y la cuarta en mujeres, según la cuenta de alto costo del Ministerio de Salud y Protección Social (Minsalud) ${ }^{5}$ y del Instituto Nacional de Cancerología ${ }^{6}$, que reportaron 3.044 casos incidentes en hombres y 1.983 en mujeres, con un incremento aproximado del $27 \%$ entre 2000 y $2011^{5-7}$.
El CP se clasifica en dos subtipos histológicos: el CP de células pequeñas (CPCP), que representa el 15\% de los casos; y el CP de células no pequeñas (CPCNP), que constituye el $85 \%$ de los $\operatorname{casos}^{1,8}$. La sobrevida global reportada a cinco años para el cáncer de pulmón en los Estados Unidos entre 2001 a 2007 fue del 15,6\% en enfermedad localizada, y aproximadamente el 52\% de los pacientes con metástasis a distancia presentaron una sobrevida a tres años del 3,6\%. Estos valores hacen imperante la necesidad de un diagnóstico oportuno para iniciar tratamiento temprano e incrementar la SLP de estos pacientes ${ }^{1}$. Afortunadamente, durante los últimos años han surgido nuevas tecnologías para el diagnóstico y el tratamiento de la enfermedad, se ha avanzado en el conocimiento del perfil genómico de la neoplasia y esto ha permitido personalizar el tratamiento y ofrecer terapias blanco dirigidas. Estos avances, junto con la utilización de la inmunoterapia, han impactado considerablemente la sobrevida de los pacientes y han cambiado el panorama del cáncer de pulmón ${ }^{9}$.

En Colombia se han realizado esfuerzos académicos para consolidar y evaluar la evidencia disponible con el fin de presentar a los profesionales de la salud las mejores herramientas para el diagnóstico, tratamiento y seguimiento de los pacientes con CP. Sin embargo, el conocimiento y la adherencia a estos documentos guía no ha sido el esperado, lo cual se ve reflejado en la variabilidad de tratamientos para una misma enfermedad. De acuerdo con lo anterior, desde la Asociación Colombiana de Hematología y 
Oncología (ACHO), con la intención de facilitar la práctica clínica, se decidió utilizar la estrategia de consenso de expertos. En consecuencia, se desarrolló este manuscrito como una alternativa asequible de revisión de la literatura, para contextualizarla conforme con las características propias del sistema de salud colombiano, en cuanto al acceso y disponibilidad de tecnologías, pretendiendo homogeneizar la conducta médica a partir de la práctica de la 'vida real' de un grupo de expertos en oncología en el tratamiento de CPCNP. Este consenso ofrece una serie de recomendaciones basadas en la mejor evidencia científica para apoyar la práctica clínica y podrá ser utilizado como un documento guía para la toma de decisiones clínicas en el tratamiento de CPCNP.

Finalmente, este trabajo es una iniciativa científica, no regulatoria, donde se incluyeron tratamientos e indicaciones aún no aprobados en Colombia, apoyados por estudios clínicos reconocidos por los expertos e incluidos en guías de manejo de oncología reconocidas internacionalmente. Se espera que estos sean aprobados próximamente por parte del Invima para que puedan ser ofrecidos y suministrados a todos los pacientes que lo requieran.

De acuerdo con ello, el objetivo general de este proyecto fue consensuar por común acuerdo de expertos recomendaciones para el manejo del cáncer de pulmón metastásico (estadio IV) de célula no pequeña conforme con el contexto colombiano.

\section{Consideraciones}

Teniendo en cuenta las características del sistema de salud colombiano y las disposiciones regulatorias para el uso de medicamentos dictadas por el Invima, el uso de las terapias que se mencionan en este consenso está sujeto a la disponibilidad de acuerdo con su contexto local y según indicación aprobada por el Invima. Por esto, los pacientes deberán recibir inicialmente los tratamientos aprobados y disponibles en el país.

\section{Métodos}

Se estructuró un consenso de expertos constituido por 17 oncólogos clínicos expertos en cáncer de pulmón, miembros activos de la Asociación Colombiana de Hematología y Oncología (ACHO), de 10 ciudades colombianas (Bogotá, Medellín, Cali, Bucaramanga, Manizales, Armenia, Pereira, Barranquilla, Montería y
Valledupar). Con estos se conformó un grupo desarrollador compuesto por tres oncólogos y un epidemiólogo, quienes estructuraron las preguntas y analizaron la información. Adicionalmente, el director médico de Programas Internacionales y director asociado de Oncología Global del Sylvester Comprehensive Cancer Center y la University of Miami participó en la construcción de este manuscrito.

\section{Procedimiento}

El desarrollo del consenso se llevó a cabo en cuatro fases (figura 1).

Fase 1: con base en las guías de la National Comprehensive Cancer Network (NCCN) 2018 ${ }^{10}$, que actualmente son consideradas como documento de orientación, adoptado por la ACHO, el grupo desarrollador estructuró 22 preguntas con sus respectivas opciones de respuesta. Se realizó una reunión con los expertos, donde fue explicada la metodología del consenso. Para la calificación de las opciones, se utilizó una escala de 1 a 9, donde 1 se consideró como extremadamente inapropiado o un manejo no usado, y 9 como extremadamente apropiado o es la opción de primera línea en el tratamiento. Posteriormente, se construyó la matriz en una hoja de cálculo y fue enviado un cuestionario a cada uno de los expertos.

Fase 2: una vez se recibieron las calificaciones de los expertos, la información fue analizada y se definió como consenso a las calificaciones de las respuestas con medianas de: 1 a 3 con rangos intercuartílicos (RIQ) entre 1 y 3, y de 7 a 9 con RIQ entre 7 y 9 . Esta información fue consolidada y fue enviada (respuestas enmascaradas) de nuevo a los expertos para su revisión.

Fase 3: el grupo desarrollador elaboró un segundo cuestionario donde solo fueron incluidas las preguntas y respuestas no consensuadas, el cual fue enviado a los expertos para su consideración y calificación. Una vez fueron devueltos los cuestionarios por los expertos, se consolidó la información y las respuestas que no fueron consensuadas pasaron a la fase 4 para discusión en nueva reunión.

Fase 4: en esta fase, se efectuó una reunión nominal, donde se presentaron los resultados de las calificaciones de la primera y segunda ronda, y se discutieron las preguntas en las cuales no se llegó a consenso. 


\section{FASE 1}

\begin{tabular}{|c|c|c|c|}
\hline $\begin{array}{c}\text { Construcción de } \\
\text { preguntas }\end{array}$ & PRIMERA RONDA \\
\cline { 2 - 3 } & $\begin{array}{c}\text { Envío de preguntas } \\
\text { para calificación }\end{array}$ \\
\hline
\end{tabular}

\section{FASE 2}

Consolidación de respuestas

Socialización de respuestas (enmascarada)

FASE 3

\begin{tabular}{|c|c|c|}
\hline SEGUNDA RONDA & $\begin{array}{l}\text { Envío de pregntas } \\
\text { para calificación }\end{array}$ & $\begin{array}{l}\text { Consolidación } \\
\text { de respuestas }\end{array}$ \\
\hline
\end{tabular}

FASE 4

Consolidación

de información

DISCUSIÓN NOMINAL FINAL

Figura 1. Ejecución del consenso

\section{Resultados}

Se estructuraron y evaluaron 25 preguntas relacionadas con el tratamiento de cáncer de pulmón metastásico (estadio IV) de célula no pequeña, donde participaron 17 oncólogos clínicos de la ACHO. El promedio de edad de los expertos fue de 48,6 \pm 9,4 años y la experiencia en oncología estuvo distribuida de la siguiente forma: 4 (25\%) entre 5 a 10 años, 5 (31,2\%) entre 11 y 20 años, y 7 (43,7\%) más de 21 años.

\section{Preguntas}

1. En pacientes con CPCNP metastásico, ¿cuáles son las pruebas diagnósticas moleculares y de inmunohistoquímica que se deben realizar para definir tratamiento oncológico de primera línea?

- Se recomienda medir PDL1 por inmunohistoquímica en todos los pacientes con adenocarcinomas y carcinomas escamocelulares ${ }^{11-16}$.

- En pacientes no fumadores con carcinomas escamocelulares y en todos los pacientes con adenocarcinomas, se recomienda medir EGFR, ALK, ROS1 $15-20$.
Para la medición de mutación de BRAF y otras mutaciones, no hubo consenso para llevarla a cabo de forma sistemática, dada su baja frecuencia y ausencia de terapia sistémica específica efectiva. Sin embargo, se consideró que si estas son solicitadas deben hacerse secuencialmente después de descartar las mutaciones más frecuentes.

2. En pacientes con adenocarcinoma de pulmón metastásico con EGFR, ALK, ROS1 y PD-L1 desconocidos, en quienes se inició tratamiento con quimioterapia citotóxica y se descubre mutación EGFR posterior al tercer ciclo de quimioterapia y en evaluación de respuesta, si se evidencia un paciente con enfermedad estable o respuesta parcial, ¿cuál es la mejor conducta?

- Se recomienda completar 4-6 ciclos de quimioterapia y secuencialmente iniciar anti-EGFR de mantenimiento.

- Interrumpir quimioterapia e iniciar tratamiento con anti-EGFR. 
- Completar 4-6 ciclos de quimioterapia y seguir mantenimiento con quimioterapia hasta progresión, solo en casos de buena respuesta inicial a la quimioterapia $21-23$

- No se recomienda después de 4 a 6 ciclos de quimioterapia planeada continuar mantenimiento combinado con pemetrexed o bevacizumab con anti-EGFR ${ }^{24-28}$.

Se consideró que la evidencia científica disponible para estas estrategias de mantenimiento combinado (quimioterapia más anti-EGFR) es débil y sin impacto clínicamente significativo en sobrevida, solo con un leve aumento de sobrevida libre de progresión (SLP).

3. En pacientes con adenocarcinoma de pulmón metastásico con EGFR, ALK, ROS1 y PD-L1 desconocidos, en quienes se inició tratamiento con quimioterapia citotóxica y se descubre mutación EGFR posterior al tercer ciclo y en evaluación de respuesta, si se evidencia enfermedad en progresión, ¿cuál sería la mejor conducta?

Se recomienda interrumpir el tratamiento con quimioterapia e iniciar medicamento anti-EGFR-121,29-44.

4. En pacientes con adenocarcinoma de pulmón metastásico con EGFR, ALK, ROS1 y PD-L1 desconocidos, en quienes se inició tratamiento con quimioterapia citotóxica y se descubre rearreglo de ALK posterior al tercer ciclo de quimioterapia, si se evidencia un paciente con enfermedad estable o respuesta parcial, ¿cuál es la mejor conducta?

- Se recomienda interrumpir quimioterapia e iniciar terapia blanco dirigida.

- Se aconseja completar quimioterapia de 4-6 ciclos, e iniciar terapia blanco dirigida.

- En el caso de que el paciente presente una buena respuesta y tolerancia al tratamiento, se considera que se puede continuar el tratamiento con quimioterapia hasta la progresión ${ }^{45-48}$

5. En pacientes con adenocarcinoma de pulmón metastásico con EGFR, ALK, ROS1 y PD-L1 desconocidos, en quienes se inició tratamiento con quimioterapia citotóxica y se descubre rearreglo de ALK durante quimioterapia, si se evidencia enfermedad en progresión, ¿cuál es la mejor conducta?

Se recomienda interrumpir tratamiento de quimioterapia e iniciar tratamiento blanco dirigido $44,46,49$.

6. En pacientes con adenocarcinoma de pulmón metastásico EGFR mutado, sin compromiso cerebral ECOG 0-2, ¿cuál es el tratamiento de elección en primera línea?

Se recomienda el uso de cualquiera de los siguientes medicamentos anti-EGFR $29,30,32,34,40,43,50$ :

Afatinib

Osimertinib

Gefitinib

Erlotinib

7. En pacientes con adenocarcinoma de pulmón metastásico EGFR mutado con compromiso cerebral ECOG 0-2, ¿cuál es el tratamiento de elección en primera línea?

Se recomienda como primera opción de tratamiento el uso del osimertinib, sin embargo, se puede considerar también el uso de afatinib, gefitinib o erlotinib29,30,32,34,40,43,48

8. En pacientes con cáncer de pulmón metastásico EGFR mutado en progresión durante tratamiento blanco dirigido de primera línea, ¿siempre se debería realizar una biopsia para estudio de mutación de T790 a fin de definir tratamiento sistémico por seguir?

- Se recomienda siempre ante progresión tumoral durante tratamiento anti-EGFR realizar nueva biopsia para estudio de resistencia y definir siguiente línea de tratamiento.

- Se debe considerar siempre tomar biopsia de tejido y biopsia líquida29,30.

\section{En pacientes con carcinoma de pulmón metastásico} EGFR mutado, ECOG0-2, en progresión tumoral lenta, asintomáticauoligometastásica, durantetratamiento conerlotinib,afatinibogefitinib,¿cuálseríaeltratamiento de elección?

- Se recomienda continuar con afatinib, gefitinib o erlotinib en pacientes con progresión lenta y asintomática. 
- En el caso de que exista una mutación T790, se sugiere cambiar el tratamiento a osimertinib como segunda línea. Y si la mutación T790 es negativa, considerar el cambio de tratamiento a quimioterapia.

- Es importante considerar siempre la terapia local para las lesiones oligometastásicas ${ }^{51-54}$.

10. En pacientes con carcinoma de pulmón metastásico EGFR mutado, ECOG 0-2, en progresión tumoral sintomática y enfermedad oligometastásica cerebral exclusiva, durante tratamiento con erlotinib, afatinib o gefitinib, ¿cuál es el tratamiento de elección?

- Se recomienda considerar como primera opción el inicio de osimertinib en mutación T790 positiva más administración de terapia local.

- Se considera como opción razonable en progresión cerebral exclusiva lenta y sintomática, y enfermedad oligometastásica: continuar afatinib, gefitinib o erlotinib más administración de terapia local.

- No se aconseja administrar quimioterapia en este escenario ${ }^{31-36}$

11. En pacientes con carcinoma de pulmón metastásico EGFR mutado, ECOG 0-2, en progresión tumoral sintomáticaymetástasiscerebralesmúltiples, durante tratamientoconerlotinib, afatinibogefitinib,¿cuálsería el tratamiento de elección?

- Se recomienda considerar el uso del osimertinib como segunda línea de tratamiento, si la mutación T790 es positiva, y administración de terapia local.

- En el caso de que el paciente se encuentre en adecuado estado clínico y funcional, cambiar manejo sistémico a quimioterapia.

- Siempre se debe considerar administrar terapia local.

- No se aconseja continuar afatinib, gefitinib o erlotinib $^{51-62}$.

12. En pacientes con carcinoma de pulmón metastásico, EGFR mutado, ECOG 0-2 y enfermedad en progresiónoligometastásicasintomáticanocerebral, durante tratamientoconerlotinib, afatinibogefitinib, ¿cuál es el tratamiento de elección?

- Se recomienda continuar tratamiento con afatinib, gefitinib o erlotinib, más terapia local en pacientes con enfermedad oligometastásica sintomática.
- Se debe considerar el inicio de tratamiento con osimertinib en presencia de mutación T790 y concomitantemente el uso de terapia local.

- Se considera el cambio de manejo sistémico a quimioterapia, es una opción razonable si la mutación T790 es negativa y el paciente se encuentra en adecuado estado clínico y funcional.

- Se aconseja siempre administrar terapia loca|31-34.

13. En pacientes con carcinoma de pulmón metastásico, EGFR mutado, ECOG 0-2 y enfermedad en progresión sintomática, durante tratamiento con erlotinib, afatinib o gefinitib, con lesiones metastásicas múltiples no cerebrales, ¿cuál es el tratamiento de elección?

- Se recomienda iniciar tratamiento con osimertinib si el paciente presenta la mutación T790.

- Se considera como una opción razonable iniciar quimioterapia citotóxica.

- No se aconseja continuar con erlotinib, afatinib o gefitinib en este tipo de escenarios clínicos ${ }^{32-34}$.

14. En pacientes con carcinoma escamocelular de pulmón, con metástasis cerebrales, EGFR mutado y ECOG 0-2, ¿cuál es el tratamiento de elección en primera línea?

Se recomienda el uso de cualquiera de los siguientes medicamentos anti-EGFR $29,30,32,34,40,43,63$.

Afatinib

Gefitinib

Erlotinib

Osimertinib

15. En pacientes con carcinoma de pulmón metastásico, con rearreglo ALK y ECOG 0-2, ¿cuál es el tratamiento de primera línea?

Se recomienda iniciar tratamiento con crizotinib, alectinib o ceritinib, y no se considera pertinente administrar quimioterapia citotóxica ${ }^{45-48}$.

16. En pacientes con carcinoma de pulmón metastásico, con rearreglo ROS1 y ECOG 0-2, ¿cuál es el tratamiento de elección?

- Se recomienda iniciar tratamiento con crizotinib o ceritinib ${ }^{1,64-67}$.

- Administrar quimioterapia en ausencia de terapia blanco dirigida es una opción razonable ${ }^{68,69}$. 
17. En pacientes con carcinoma de pulmón metastásico, con mutación de BRAF y ECOG 0-2, ¿cuál es el tratamiento de elección?

- Se recomienda iniciar tratamiento con dabrafenib más trametinib ${ }^{70,71}$.

- Considerar el uso de quimioterapia basada en platino.

18. En pacientes con adenocarcinoma o cáncer escamocelular de pulmón metastásico, ECOG 0-2,

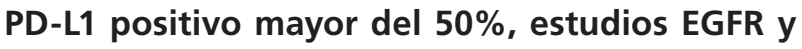
ALK negativos o desconocidos, ¿cuál es el tratamiento de elección en primera línea?

Se recomienda iniciar tratamiento con pembrolizumab en primera línea. Sin embargo, en los casos donde exista alguna contraindicación para la administración de inmunoterapia, considerar administrar quimioterapia basada en platino ${ }^{72}$.

19. En pacientes con adenocarcinoma de pulmón metastásico, ECOG 0-2, PD-L1 positivo menor del $50 \%$, estudios EGFR y ALK negativos o desconocidos, ¿cuál es el tratamiento de elección de primera línea?

- Se recomienda iniciar tratamiento con quimioterapia más inmunoterapia.

- La administración de quimioterapia basada en platino también es una opción razonable.

- No se aconseja administrar tratamiento con pembrolizumab monoagente en primera línea si PD-L1 es menor del $50 \%{ }^{73,74}$.

20. En pacientes con adenocarcinoma de pulmón metastásico, ECOG 0-2, PD-L1 positivo menor del $50 \%$, estudios EGFR y ALK negativos o desconocidos, que ha recibido de 4 a 6 ciclos de quimioterapia basada en platino en primera línea con respuesta parcial o enfermedad estable, ¿cuál es el tratamiento de elección?

- Se recomienda mantenimiento con cualquiera de los siguientes esquemas:

Pemetrexed

Bevacizumab

Pemetrexed más bevacizumab

- No se aconseja mantenimiento con gemcitabina.

- No se considera la observación como una alternativa adecuada, teniendo en cuenta la disponibilidad de terapias de segunda línea que han demostrado un impacto positivo en la SLP de estos pacientes ${ }^{75-77}$.

21. En pacientes con adenocarcinoma de pulmón metastásico, ECOG 0-2, PD-L1 positivo mayor del $1 \%$, que han recibido de 4 a 6 ciclos de quimioterapia basada en platino en primera línea y presentan enfermedad en progresión, ¿cuál es el tratamiento de elección en segunda línea?

- Se recomienda administrar inmunoterapia en segunda línea con nivolumab, atezolizumab o pembrolizumab ${ }^{78}$.

- Se considera administrar quimioterapia si existe alguna contraindicación para inmunoterapia o no son pacientes candidatos para inmunoterapia $a^{8,69,77,79,80}$.

22. En pacientes con adenocarcinoma de pulmón metastásico,ECOG0-2,PD-L1negativo(menordel1\%),que han recibido de 4 a 6 ciclos de quimioterapia basada en platino en primera línea y presentan enfermedad en progresión, ¿cuál es el tratamiento de elección en segunda línea?

- Se recomienda iniciar tratamiento de segunda línea con nivolumab o atezolizumab en pacientes que se consideren candidatos a inmunoterapia: ECOG 0 y 2 , sin rápida progresión y que no tengan síntomas severos por compromiso tumoral.

- Se debe considerar el uso de quimioterapia en pacientes con alguna contraindicación para inmunoterapia o que no sean candidatos para esta.

- No se aconseja administrar pembrolizumab como tratamiento de segunda línea en pacientes con PDL1 negativo $8,11,68,69,78-80$.

23. En pacientes con carcinoma escamocelular de pulmón metastásico, ECOG 0-2,PD-L1 menordel $50 \%$ o desconocido, estudios EGFR y ALK negativos o desconocidos, ¿eltratamientodeelecciónen primeralínea es la quimioterapia basada en platino?

Consenso a favor en iniciar tratamiento con quimioterapia de primera línea basada en platino, por común acuerdo ${ }^{72,73}$.

24. En pacientes con carcinoma escamocelular metastásico, ECOG 0-2, con PD-L1 positivo menor 
del $50 \%$ o desconocido, estudios EGFR y ALK negativos o desconocidos, que han recibido de 4 a 6 ciclos de quimioterapia basada en platino en primera línea, con enfermedad estable, ¿cuál es el tratamiento de elección?

- Se sugiere mantener observación como manejo de elección.

- No se recomienda administrar cimavax, pues la evidencia no es sólida y no está incluido en ninguna guía de práctica clínica. De la misma forma, no se aconseja mantenimiento con quimioterapia (gemcitabina-docetaxel), pues tampoco hay evidencia que soporte su efectividad ${ }^{74-77}$.

25. En pacientes con carcinoma escamocelular metastásico, ECOG 0-2, PD-L1 positivo menor del $\mathbf{5 0 \%}$ o desconocido, estudios EGFR y ALK negativos o desconocidos, que han recibido de 4 a 6 ciclos de quimioterapia basada en platino en primera línea, con enfermedad en progresión, ¿cuál es el tratamiento de elección en segunda línea?

- Se recomienda iniciar tratamiento de segunda línea con nivolumab, pembrolizumab o atezolizumab.

- Se debe considerar tratamiento con quimioterapia en pacientes que tengan alguna contraindicación para inmunoterapia o que no sean candidatos para esta.

- No hubo consenso en cuanto a la indicación para administrar tratamiento con ramucirumab más docetaxe $\left.\right|^{8,11,68,69,78-80}$.

\section{Glosario}

EGFR: receptor del factor de crecimiento epidérmico ${ }^{81}$.

ALK: proteína quinasa de linfoma anaplásico ${ }^{81}$.

ROS1: receptor tirosina quinasa ${ }^{82}$.

PD-L1: ligando de proteína de muerte celular programada $1^{83}$

BRAF: mediador de vía proteínas quinasas activadas por mitógenos (MAPK) ${ }^{84}$.

Mutación T790: mutación específica del gen EGFR sensible a osimertinib ${ }^{81}$.

ECOG: escala de calidad de vida en pacientes con cáncer Eastern Cooperative Oncology Group ${ }^{85}$.

SLP: sobrevida libre de progresión.

\section{Referencias}

1. Arrieta O, Guzmán-De Alba E, López LF, Acosta-Espinoza A, Latorre-Alexander J, Meza JF, et al. Consenso nacional de diagnóstico y tratamiento del cáncer de pulmón de células no pequeñas. Revista de Investigación Clínica. 2013;5(S1):5-84

2. Globocan. Lung cancer estimated incidence, mortality and prevalence worldwide in 2012. [internet]. Francia; 2012 [citado 2018]. Disponible en: http://globocan.iarc.fr/Pages/fact_ sheets cancer.aspx

3. Instituto Nacional de Cáncer. Cancer statistics review, 19752002. EE. UU.; 2007 [citado 2018]. Disponible en: https://seer. cancer.gov/archive/csr/1975_2002/

4. American Cancer Society. Cáncer de pulmón de células no pequeña: estadísticas, EE. UU. Cancer.net [internet]. 2017. [citado 2018]. Disponible en https://www.cancer.net/es/ tipos-de-c\%C3\%A1ncer/c\%C3\%A1ncer-de-pulm\%C3\%B3nc\%C3\%A9lulas-no-peque\%C3\%B1as/estad\%C3\%ADsticas

5. Ministerio de Salud y Protección Social de Colombia. Guía de práctica clínica para la detección temprana, diagnóstico, estadificación y tratamiento del cáncer de pulmón. Guía para profesionales de la salud. [internet]. Colombia; 2014. [citado 2018]. Disponible en http://gpc.minsalud.gov.co/gpc_sites/repositorio/forms/allitems.aspx?rootfolder=/gpc_sites/repositorio/ conv_563/gpc_c_pulmon\&folderctid=0x012000f43cfde8d81 54047b4b4b9b88e72b8b7\&view=\%7Bc81f0233-558c-49afa456-55fa1462ce27\%7D

6. Instituto Nacional de Cancerología. Análisis de situación de cáncer en Colombia 2015 [internet]. [citado 2018]. Disponible en https://www.google.com/url?sa=t\&rct=j\&q=\&esrc $=s \&$ source $=$ web $\& c d=1 \&$ ved $=0$ ahUKEwiV96a48fXbAhWOv 1MKHSb-IYQFggnMAA\&url=http\%3A\%2F\%2F www.cancer.gov.co\%2FSituacion_del_Cancer_en_Colombia_2015. pdf\&usg=AOvVaw17RZT_CxhHpz3dbWC_tzQ6

7. Cuenta de Alto Costo-Fondo Colombiano de Enfermedades de Alto Costo. Indicadores de gestión del riesgo en pacientes con cáncer de pulmón. Colombia; 2018. [citado 2018]. Disponible en https://www.google.com/url?sa=t\&rct=j\&q=\&esr $c=s \&$ source $=$ web \&cd $=21 \&$ ved $=0$ ahUKEwibu-blgO3aAhWC 7 FMKHSsDA9U4ChAWCFcwCg\&url=https \%3A \% 2F\%2Fcue ntadealtocosto.org\%2Fsite \%2Fimages \%2FIndicadores_de_ gestion_del_riesgo_en_pacientes_con_Cancer_de_Pulmon. pdf\&usg=AOvVaw38adFfKnJX8glkn-GmDcnm

8. Castro C, Cardona AF, Revéiz L, Serrano SJ, Carranza H, Vargas CA, et al. Cáncer de pulmón de célula no pequeña metastásico. Tratamiento actual basado en la evidencia (OncolGroup). Acta Médica Colombiana. 2010;35:53-81

9. Gettinger S. Immunotherapy of advanced non-small cell lung cancer with immune checkpoint inhibition. UpToDay [internet]. 2018. [citado 2018]. Disponible en: https://uptoday/contents/ immunotherapy-of-advanced-non-small-cell-lung-cancer-withimmune-checkpoint-inhibition?search=inmunotherapy lung cancer\&source=search_result\&selectedTitle $=1 \sim 150 \&$ usage type $=$ default\&display_rank $=1$

10. National Comprehensive Cancer Network. Clinical practice guidelines in oncology. Non-small cell lung cancer. [internet]. EE. UU.; 2018. [citado 2018]. Disponible en: https://www.nccn.org/ professionals/physician_gls/default.aspx\#nscl

11. Herbst RS, Baas P, Kim DW, Felip E, Pérez-Gracia JL, Han $J Y$, et al. Pembrolizumab versus docetaxel for previously treated, PD-L1-positive, advanced non-small-cell lung can- 
cer (keynote-010): a randomised controlled trial. Lancet. 2016;387(10027):1540-50

12. Barlesi F, Mazieres J, Merlio JP, Debieuvre D, Mosser J, Lena H, et al. Routine molecular profiling of patients with advanced non-small-cell lung cancer: results of a 1-year nationwide programme of the French Cooperative Thoracic Intergroup (IFCT). Lancet. 2016;387(10026):1415-26.

13. Kris MG, Johnson BE, Berry LD, Kwiatkowski DJ, lafrate AJ, Wistuba II, et al. Using multiplexed assays of oncogenic drivers in lung cancers to select targeted drugs. JAMA. 2014;311(19):1998-2006.

14. Leighl NB, Rekhtman N, Biermann WA, Huang J, Mino-Kenudson $\mathrm{M}$, Ramalingam SS, et al. Molecular testing for selection of patients with lung cancer for epidermal growth factor receptor and anaplastic lymphoma kinase tyrosine kinase inhibitors: American Society of Clinical Oncology endorsement of the College of American Pathologists/International Association for the study of lung cancer/association for molecular pathology guideline. J Clin Oncol. 2014;32(32):3673-9.

15. Lindeman NI, Cagle PT, Beasley MB, Chitale DA, Dacic S, Giaccone $G$, et al. Molecular testing guideline for selection of lung cancer patients for EGFR and ALK tyrosine kinase inhibitors: guideline from the College of American Pathologists, International Association for the Study of Lung Cancer, and Association for Molecular Pathology. Arch Pathol Lab Med. 2013;137(6):828-60.

16. Bergethon K, Shaw AT, Ou SH, Katayama R, Lovly CM, McDonald NT, et al. ROS1 rearrangements define a unique molecular class of lung cancers. J Clin Oncol. 2012;30(8):863-70.

17. Lindeman $\mathrm{NI}$, Cagle PT, Aisner DL, Arcila ME, Beasley MB, Bernicker $\mathrm{EH}$, et al. Updated molecular testing guideline for the selection of lung cancer patients for treatment with targeted tyrosine kinase inhibitors: guideline from the College of American Pathologists, the International Association for the Study of Lung Cancer, and the Association for Molecular Pathology. Arch Pathol Lab Med. 2018;142(3):321-46.

18. Lindeman NI, Cagle PT, Beasley MB, Chitale DA, Dacic S, Giaccone $G$, et al. Molecular testing guideline for selection of lung cancer patients for EGFR and ALK tyrosine kinase inhibitors: guideline from the College of American Pathologists, International Association for the Study of Lung Cancer, and Association for Molecular Pathology. J Thorac Oncol. 2013;8(7):823-59.

19. Sacher AG, Paweletz C, Dahlberg SE, Alden RS, O'Connell A, Feeney $\mathrm{N}$, et al. Prospective validation of rapid plasma genotyping for the detection of EGFR and KRAS mutations in advanced lung cancer. JAMA Oncol. 2016;2(8):1014-22.

20. Wu YL, Sequist LV, Hu CP, Feng J, Lu S, Huang $Y$, et al. EGFR mutation detection in circulating cell-free DNA of lung adenocarcinoma patients: analysis of LUX-Lung 3 and 6 . Br J Cancer. 2017;116(2):175-85.

21. Gridelli C, Ciardiello F, Gallo C, Feld R, Butts C, Gebbia V, et al. First-line erlotinib followed by second-line cisplatin-gemcitabine chemotherapy in advanced non-small-cell lung cancer: the TORCH randomized trial. J Clin Oncol. 2012;30(24):3002-11.

22. Brugger W, Triller N, Blasinska-Morawiec M, Curescu S, Sakalauskas R, Manikhas GM, et al. Prospective molecular marker analyses of EGFR and KRAS from a randomized, placebo-controlled study of erlotinib maintenance therapy in advanced non-small-cell lung cancer. J Clin Oncol. 2011:29(31):4113-20.
23. Cappuzzo F, Ciuleanu T, Stelmakh L, Cicenas S, Szczésna $A$, Juhasz $E$, et al. Erlotinib as maintenance treatment in advanced non-small-cell lung cancer: a multicentre, randomized, placebo-controlled phase 3 study. Lancet Oncol. 2010;11(6):521-9.

24. Rosell R, Dafni U, Felip E, Curioni-Fontecedro A, Gautschi O, Peters $S$, et al. Erlotinib and bevacizumab in patients with advanced non-small-cell lung cancer and activating EGFR mutations (BELIEF): an international, multicentre, single-arm, phase 2 trial. Lancet Respir Med. 2017;5(5):435-44.

25. Seto $T$, Kato $T$, Nishio $M$, Goto $K$, Atagi $S$, Hosomi $Y$, et al. Erlotinib alone or with bevacizumab as first-line therapy in patients with advanced non-squamous non-small-cell lung cancer harbouring EGFR mutations (JO25567): an open-label, randomised, multicentre, phase 2 study. Lancet Oncol. 2014;15(11):1236-44.

26. Herbst RS, Prager $D$, Hermann $R$, Fehrenbacher $L$, Johnson $B E$, Sandler A, et al. TRIBUTE: a phase III trial of erlotinib hydrochloride (OSI-774) combined with carboplatin and paclitaxel chemotherapy in advanced non-small-cell lung cancer. J Clin Oncol. 2005;23(25):5892-9.

27. Giaccone G, Herbst RS, Manegold C, Scagliotti G, Rosell R, Miller $V$, et al. Gefitinib in combination with gemcitabine and cisplatin in advanced non-small-cell lung cancer: a phase III trial-INTACT 1. J Clin Oncol. 2004;22(5):777-84

28. Herbst RS, Giaccone G, Schiller JH, Natale RB, Miller V, Manegold $C$, et al. Gefitinib in combination with paclitaxel and carboplatin in advanced non-small-cell lung cancer: a phase III trial--INTACT 2. J Clin Oncol. 2004;22(5):785-94.

29. Zhou C, Wu YL, Chen G, Feng J, Liu XQ, Wang C, et al. Final overall survival results from a randomised, phase III study of erlotinib versus chemotherapy as first-line treatment of EGFR mutation-positive advanced non-smallcell lung cancer (OPTIMAL, CTONG-0802). Ann Oncol. 2015;26(9):1877-83

30. Rosell R, Carcereny E, Gervais R, Vergnenegre A, Massuti B, Felip $E$, et al. Erlotinib versus standard chemotherapy as firstline treatment for European patients with advanced EGFR mutation-positive non-small-cell lung cancer (EURTAC): a multicentre, open-label, randomized phase 3 trial. Lancet Oncol. 2012;13(3):239-46.

31. Wu YL, Zhou C, Liam CK, Wu G, Liu X, Zhong Z, et al. First-line erlotinib versus gemcitabine/cisplatin in patients with advanced EGFR mutation-positive non-small-cell lung cancer: analyses from the phase III, randomized, open-label, ENSURE study. Ann Oncol. 2015;26(9):1883-9.

32. Mok TS, Wu YL, Thongprasert $S$, Yang CH, Chu DT, Saijo N, et al. Gefitinib or carboplatin-paclitaxel in pulmonary adenocarcinoma. N Engl J Med. 2009;361(10):947-57.

33. Fukuoka $M, W u Y L$, Thongprasert $S$, Sunpaweravong $P$, Leong SS, Sriuranpong $V$, et al. Biomarker analyses and final overall survival results from a phase III, randomized, open-label, firstline study of gefitinib versus carboplatin/paclitaxel in clinically selected patients with advanced non-small-cell lung cancer in Asia (IPASS). J Clin Oncol. 2011;29(21):2866-74.

34. Mitsudomi T, Morita S, Yatabe $Y$, Negoro S, Okamoto I, Tsurutani J, et al. Gefitinib versus cisplatin plus docetaxel in patients with non-small-cell lung cancer harbouring mutations of the epidermal growth factor receptor (WJTOG3405): an open label, randomised phase 3 trial. Lancet Oncol. 2010;11(2):121-8. 
35. Inoue A, Kobayashi K, Maemondo M, Sugawara S, Oizumi S, Isobe $\mathrm{H}$, et al. Updated overall survival results from a randomized phase III trial comparing gefitinib with carboplatin-paclitaxel for chemo-naïve non-small cell lung cancer with sensitive EGFR gene mutations (NEJ002). Ann Oncol. 2013;24(1):54-9.

36. Maemondo $M$, Inoue A, Kobayashi K, Sugawara S, Oizumi S, Isobe H, et al. Gefitinib or chemotherapy for nonsmall-cell lung cancer with mutated EGFR. N Engl J Med. 2010;362(25):2380-8

37. Oizumi S, Kobayashi $K$, Inoue $A$, Maemondo $M$, Sugawara $\mathrm{S}$, Yoshizawa $\mathrm{H}$, et al. Quality of life with gefitinib in patients with EGFR-mutated non-small cell lung cancer: quality of life analysis of North East Japan Study Group 002 Trial. Oncologist. 2012;17(6):863-70.

38. Miller VA, Hirsh V, Cadranel J, Chen YM, Park K, Kim SW, et al Afatinib versus placebo for patients with advanced, metastatic non-small-cell lung cancer after failure of erlotinib, gefitinib, or both, and one or two lines of chemotherapy (LUX-Lung 1): a phase 2b/3 randomised trial. Lancet Oncol. 2012;13(5):528-38.

39. $\mathrm{Wu} Y L$, Zhou $C$, Hu C-P, Feng JF, Lu S, Huang $Y$, et al. LUXLung 6: a randomized, open-label, phase III study of afatinib (A) versus gemcitabine/cisplatin (GC) as first-line treatment for Asian patients (pts) with EGFR mutation-positive (EGFR M+) advanced adenocarcinoma of the lung. J Clin Oncol. 2013;31(15 suppl):8016.

40. Sequist LV, Yang JC, Yamamoto N, O’Byrne K, Hirsh V, Mok T, et al. Phase III study of afatinib or cisplatin plus pemetrexed in patients with metastatic lung adenocarcinoma with EGFR mutations. J Clin Oncol. 2013;31(27):3327-34.

41. Yang JC, Hirsh V, Schuler M, Yamamoto N, O'Byrne KJ, Mok TS, et al. Symptom control and quality of life in LUX-Lung 3: a phase III study of afatinib or cisplatin/pemetrexed in patients with advanced lung adenocarcinoma with EGFR mutations. J Clin Oncol. 2013;31(27):3342-50

42. Katakami N, Atagi S, Goto K, Hida T, Horai T, Inoue A, et al LUX-Lung 4: a phase II trial of afatinib in patients with advanced non-small-cell lung cancer who progressed during prior treatment with erlotinib, gefitinib, or both. J Clin Oncol. 2013;31(27):3335-41.

43. Wu YL, Zhou C, Hu CP, Feng J, Lu S, Huang Y, et al. Afatinib versus cisplatin plus gemcitabine for first-line treatment of Asian patients with advanced non-small-cell lung cancer harbouring EGFR mutations (LUX-Lung 6): an open-label, randomized phase 3 trial. Lancet Oncol. 2014;15(2):213-22.

44. Food and Drug Administration. Approved drug products, 2018. [internet]. EE. UU.; 2018. [citado 2018]. Disponible en https://www.accessdata.fda.gov/scripts/cder/daf/index. $\mathrm{cfm}$ ?event=reportsSearch. . process]

45. Hida $T$, Nokihara $H$, Kondo $M$, Kim $Y H$, Azuma $K$, Seto $T$, et al. Alectinib versus crizotinib in patients with ALK-positive nonsmall-cell lung cancer (J-ALEX): an open-label, randomised phase 3 trial. Lancet. 2017;390(10089):29-39.

46. Peters $S$, Camidge DR, Shaw AT, Gadgeel S, Ahn JS, Kim DW, et al. Alectinib versus crizotinib in untreated ALK-positive nonsmall-cell lung cancer. N Engl J Med. 2017;377(9):829-38.

47. Solomon BJ, Mok T, Kim DW, Wu YL, Nakagawa $K$, Mekhail $T$, et al. First-line crizotinib versus chemotherapy in ALK-positive lung cancer. N Engl J Med. 2014;371(23):2167-77.

48. Soria JC, Tan DSW, Chiari R, Wu YL, Paz-Ares L, Wolf J, et al. First-line ceritinib versus platinum-based chemotherapy in advanced ALK-rearranged non-small-cell lung cancer (ASCEND-4): a randomized, open-label, phase 3 study. Lancet. 2017;389(10072):917-29.

49. Ou SH, Jänne PA, Bartlett $C H$, Tang $Y$, Kim DW, Otterson GA et al. Clinical benefit of continuing ALK inhibition with crizotinib beyond initial disease progression in patients with advanced ALK-positive NSCLC. Ann Oncol. 2014;25(2):415-22.

50. Soria JC, Ohe Y, Vansteenkiste J, Reungwetwattana T, Chewaskulyong $\mathrm{B}$, Lee $\mathrm{KH}$, et al. Osimertinib in untreated EGFRmutated advanced non-small-cell lung cancer. $N$ Engl J Med. 2018;378(2):113-25

51. Nishie K, Kawaguchi T, Tamiya A, Mimori T, Takeuchi N, Matsuda $Y$, et al. Epidermal growth factor receptor tyrosine kinase inhibitors beyond progressive disease: a retrospective analysis for Japanese patients with activating EGFR mutations. J Thorac Oncol. 2012;7(11):1722-7.

52. Jänne PA, Yang JC, Kim DW, Planchard D, Ohe Y, Ramalingam SS, et al. AZD9291 in EGFR inhibitor-resistant non-small-cell lung cancer. N Engl J Med. 2015;372(18):1689-99.

53. Soria JC, Wu YL, Nakagawa K, Kim SW, Yang JJ, Ahn MJ, et al. Gefitinib plus chemotherapy versus placebo plus chemotherapy in EGFR-mutation-positive non-small-cell lung cancer after progression on first-line gefitinib (IMPRESS): a phase 3 randomized trial. Lancet Oncol. 2015;16(8):990-8.

54. Yang JC, Ahn MJ, Kim DW, Ramalingam SS, Sequist LV, Su WC, et al. Osimertinib in pretreated T790M-positive advanced nonsmall-cell lung cancer: AURA study phase II extension component. J Clin Oncol. 2017;35(12):1288-96.

55. Andrews DW, Scott $C B$, Sperduto PW, Flanders AE, Gaspar LE, Schell MC, et al. Whole brain radiation therapy with or without stereotactic radiosurgery boost for patients with one to three brain metastases: phase III results of the RTOG 9508 randomized trial. Lancet. 2004;363(9422):1665-72

56. Kondziolka D, Patel A, Lunsford LD, Kassam A, Flickinger JC. Stereotactic radiosurgery plus whole brain radiotherapy versus radiotherapy alone for patients with multiple brain metastases. Int J Radiat Oncol Biol Phys. 1999;45(2):427-34.

57. Zhou L, He J, Xiong W, Liu Y, Xiang J, Yu Q, et al. Impact of whole brain radiation therapy on CSF penetration ability of icotinib in EGFR-mutated non-small cell lung cancer patients with brain metastases: results of phase I dose-escalation study. Lung Cancer. 2016;96:93-100

58. Zeng YD, Liao $H$, Qin $T$, Zhang $L$, Wei WD, Liang JZ, et al. Blood-brain barrier permeability of gefitinib in patients with brain metastases from non-small-cell lung cancer before and during whole brain radiation therapy. Oncotarget. 2015;6(10):8366-76

59. Deng $Y$, Feng $W, W u$ J, Chen $Z$, Tang $Y$, Zhang $H$, et al. The concentration of erlotinib in the cerebrospinal fluid of patients with brain metastasis from non-small-cell lung cancer. Mol Clin Oncol. 2014;2(1):116-20.

60. Togashi $Y$, Masago K, Fukudo M, Terada T, Fujita S, Irisa K, et al. Cerebrospinal fluid concentration of erlotinib and its active metabolite OSI-420 in patients with central nervous system metastases of non-small cell lung cancer. J Thorac Oncol. 2010;5(7):950-5

61. Tamiya A, Tamiya M, Nishihara $T$, Shiroyama T, Nakao K, Tsuji T, et al. 1241P - Afatinib efficacy and cerebrospinal fluid concentration in NSCLC patients with EGFR mutation developing leptomeningeal carcinomatosis. ESMO 2016 Congress. [internet]. 
2016 [citado 2018]. Disponible en: http://oncologypro.esmo. org/Meeting-Resources/ESMO-2016/Afatinib-efficacy-and-cerebrospinal-fluid-concentration-in-NSCLC-patients-with-EGFRmutation-developing-leptomeningeal-carcinomatosis

62. Ballard P, Yates JW, Yang Z, Kim DW, Yang JC, Cantarini M, et al. Preclinical comparison of osimertinib with other EGFRTKIs in EGFR-mutant NSCLC brain metastases models, and early evidence of clinical brain metastases activity. Clin Cancer Res. 2016;22(20):5130-40

63. Soria JC, Ohe Y, Vansteenkiste J, Reungwetwattana T, Chewaskulyong $\mathrm{B}$, Lee $\mathrm{KH}$, et al. Osimertinib in untreated EGFRmutated advanced non-small-cell lung cancer. N Engl J Med. 2018;378(2):113-25.

64. Shaw AT, Ou SH, Bang YJ, Camidge DR, Solomon BJ, Salgia R, et al. Crizotinib in ROS1-rearranged non-small-cell lung cancer. N Engl J Med. 2014;371(21):1963-71.

65. Wu YL, Yang JC, Kim DW, Lu S, Zhou J, Seto T, et al. Phase II study of crizotinib in East Asian patients with ROS1positive advanced non-small-cell lung cancer. J Clin Oncol. 2018;36(14):1405-11.

66. Lim SM, Kim HR, Lee JS, Lee KH, Lee YG, Min YJ, et al. Openlabel, multicenter, phase II study of ceritinib in patients with non-small-cell lung cancer harboring ROS1 rearrangement. J Clin Oncol. 2017;35(23):2613-8.

67. Drilon A, Somwar R, Wagner JP, Vellore NA, Eide CA, Zabriskie $M S$, et al. A novel crizotinib-resistant solvent-front mutation responsive to cabozantinib therapy in a patient with ROS1rearranged lung cancer. Clin Cancer Res. 2016;22(10):2351-8.

68. Garon EB, Rizvi NA, Hui R, Leighl N, Balmanoukian AS, Eder JP, et al. Pembrolizumab for the treatment of non-small-cell lung cancer. N Engl J Med. 2015;372(21):2018-28.

69. Barlesi F, Scherpereel A, Rittmeyer A, Pazzola A, Ferrer Tur N, $\mathrm{Kim} \mathrm{JH}$, et al. Randomized phase III trial of maintenance bevacizumab with or without pemetrexed after first-line induction with bevacizumab, cisplatin, and pemetrexed in advanced nonsquamous non-small-cell lung cancer: AVAPERL (MO22089). J Clin Oncol. 2013;31(24):3004-11.

70. Planchard D, Smit EF, Groen HJM, Mazieres J, Besse B, HeIland $A$, et al. Dabrafenib plus trametinib in patients with previously untreated BRAF(V600E)-mutant metastatic non-smallcell lung cancer: an open-label, phase 2 trial. Lancet Oncol. 2017;18(10):1307-16

71. Planchard D, Besse B, Groen HJM, Souquet PJ, Quoix E, Baik $C S$, et al. Dabrafenib plus trametinib in patients with previously treated BRAF(V600E)-mutant metastatic non-small cell lung cancer: an open-label, multicentre phase 2 trial. Lancet Oncol. 2016;17(7):984-93.

72. Delbaldo C, Michiels S, Syz N, Soria JC, Le Chevalier T, Pignon JP. Benefits of adding a drug to a single-agent or a 2-agent chemotherapy regimen in advanced non-small-cell lung cancer: a meta-analysis. JAMA. 2004;292(4):470-84.

73. Ardizzoni A, Boni L, Tiseo M, Fossella FV, Schiller JH, Paesmans $M$, et al. Cisplatin- versus carboplatin-based chemotherapy in first-line treatment of advanced non-small-cell lung cancer: an individual patient data meta-analysis. J Natl Cancer Inst. 2007;99(11):847-57.
74. Fidias PM, Dakhil SR, Lyss AP, Loesch DM, Waterhouse DM, Bromund JL, et al. Phase III study of immediate compared with delayed docetaxel after front-line therapy with gemcitabine plus carboplatin in advanced non-small-cell lung cancer. J Clin Oncol. 2009;27(4):591-8.

75. Pérol M, Chouaid C, Pérol D, Barlési F, Gervais R, Westeel V, et al. Randomized, phase III study of gemcitabine or erlotinib maintenance therapy versus observation, with predefined second-line treatment, after cisplatin-gemcitabine induction chemotherapy in advanced non-small-cell lung cancer. J Clin Oncol. 2012;30(28):3516-24.

76. Rodríguez PC, Neninger E, García B, Popa X, Viada C, Luaces $P$, et al. Safety, immunogenicity and preliminary efficacy of multiple-site vaccination with an epidermal growth factor (EGF) based cancer vaccine in advanced non-small cell lung cancer (NSCLC) patients. J Immune Based Ther Vaccines. 2011;9:7.

77. Rodríguez PC, Popa X, Martínez O, Mendoza S, Santiesteban E, Crespo T, et al. A phase III clinical trial of the epidermal growth factor vaccine CIMAvax-EGF as switch maintenance therapy in advanced non-small cell lung cancer patients. Clin Cancer Res. 2016;22(15):3782-90

78. Horn L, Spigel DR, Vokes EE, Holgado E, Ready N, Steins $M$, et al. Nivolumab versus docetaxel in previously treated patients with advanced non-small-cell lung cancer: twoyear outcomes from two randomized, open-label, phase III trials (CheckMate 017 and CheckMate 057). J Clin Oncol. 2017;35(35):3924-33.

79. Brahmer J, Reckamp KL, Baas $P$, Crino L, Eberhardt WE, Poddubskaya $E$, et al. Nivolumab versus docetaxel in advanced squamous-cell non-small-cell lung cancer. N Engl J Med. 2015;373(2):123-35.

80. Peters $S$, Gettinger $S$, Johnson $M L$, Jänne $P A$, Garassino $M C$, Christoph D, et al. Phase II trial of atezolizumab as first-line or subsequent therapy for patients with programmed deathligand 1-selected advanced non-small-cell lung cancer (BIRCH). J Clin Oncol. 2017;35(24):2781-9.

81. American Cancer Society. Medicamentos de terapia dirigida para el cáncer de pulmón no microcítico. [internet]. EE. UU.; 2017 [citado 2018]. Disponible en: https://www.cancer.org/es/ cancer/cancer-de-pulmon-no-microcitico/tratamiento/terapiasdirigidas.html

82. National Center for Biotechnology Information. ROS1 ROS proto-oncogene 1, receptor tyrosine kinase [Homo sapiens (human)]. [internet]. EE. UU.; 2018 [citado 2018]. Disponible en: https://www.ncbi.nlm.nih.gov/gene/6098

83. National Center for Biotechnology Information. Programmed cell death 1 ligand 1. [internet]. EE. UU.; 2017 [citado 2018]. Disponible en: https://www.ncbi.nlm.nih.gov/gene/100196322

84. Genetics Home Reference - National Library of Medicine. B-RAF proto-oncogene, serine/threonine kinase. [internet]. EE. UU.; 2017 [citado 2018]. Disponible en: https://ghr.nlm.nih.gov/ gene/BRAF

85. Eastern Cooperative Oncology Group. ECOG performance status. [internet]. EE. UU.; 2018 [citado 2018]. Disponible en: http://ecog-acrin.org/resources/ecog-performance-status 